\title{
In silico prediction of the granzyme B degradome
}

\author{
Lawrence JK Wee ${ }^{1 *}$, Esmond PS Er ${ }^{1}$, Lisa FP Ng${ }^{2,3}$, JC Tong ${ }^{1,3}$
}

From Asia Pacific Bioinformatics Network (APBioNet) Tenth International Conference on Bioinformatics - First

ISCB Asia Joint Conference 2011 (InCoB/ISCB-Asia 2011)

Kuala Lumpur, Malaysia. 30 November - 2 December 2011

\begin{abstract}
Background: Granzyme B is a serine protease which cleaves at unique tetrapeptide sequences. It is involved in several signaling cross-talks with caspases and functions as a pivotal mediator in a broad range of cellular processes such as apoptosis and inflammation. The granzyme B degradome constitutes proteins from a myriad of functional classes with many more expected to be discovered. However, the experimental discovery and validation of bona fide granzyme B substrates require time consuming and laborious efforts. As such, computational methods for the prediction of substrates would be immensely helpful.

Results: We have compiled a dataset of 580 experimentally verified granzyme B cleavage sites and found distinctive patterns of residue conservation and position-specific residue propensities which could be useful for in silico prediction using machine learning algorithms. We trained a series of support vector machines (SVM) classifiers employing Bayes Feature Extraction to predict cleavage sites using sequence windows of diverse lengths and compositions. The SVM classifiers achieved accuracy and $A_{\text {ROC }}$ scores between $71.00 \%$ to $86.50 \%$ and 0.78 to 0.94 respectively on independent test sets. We have applied our prediction method on the Chikungunya viral proteome and identified several regulatory domains of viral proteins to be potential sites of granzyme B cleavage, suggesting direct antiviral activity of granzyme B during host-viral innate immune responses.
\end{abstract}

Conclusions: We have compiled a comprehensive dataset of granzyme B cleavage sites and developed an accurate SVM-based prediction method utilizing Bayes Feature Extraction to identify novel substrates of granzyme $\mathrm{B}$ in silico. The prediction server is available online, together with reference datasets and supplementary materials.

\section{Background}

Proteolysis - the specific and limited cleavage of proteins by enzymes called proteases - represents an important mechanism for post-translational control in all living organisms [1]. Granzymes (short for granule enzymes) belong to a unique class of serine proteases which are known to mediate critical roles in the innate immune response against virus-infected or tumor cells through the induction of apoptotic cell death [2]. Consequently, the enzymes have been implicated in the pathogenesis of several chronic inflammatory and cardiovascular disorders. Granzymes are released into the cytoplasm of the target cells through endocytosis of cytolytic granules

\footnotetext{
* Correspondence: lawrence@bic.nus.edu.sg

'Data Mining Department, Institute for Infocomm Research, 1 Fusionopolis

Way, \#21-01 Connexis South Tower, Singapore 138632

Full list of author information is available at the end of the article
}

released by cytotoxic $\mathrm{T}$ cells or natural killer cells [2]. Once released into the target cells, granzymes go on to cleave specific cellular proteins and activate multiple signaling pathways leading to apoptotic cell death. Of the five human subtypes discovered to date (granzymes A, $B, H, K$ and $M$ ), granzyme $B$ has been the most well studied. Like caspases, granzyme $B$ recognizes specific tetrapeptide sequence motifs $\left(\mathrm{P}_{4}-\mathrm{P}_{3}-\mathrm{P}_{2}-\mathrm{P}_{1}\right)$ and cleave proteins after aspartate residue at $\mathrm{P}_{1}[3,4]$. Besides cleaving specific proteins regulating apoptotic cell death, granzyme B has been reported to cleave proteins across a wide spectrum of other functional classes, ranging from nuclear and cytoskeletal components to membrane receptors and viral proteins [5].

To date, more than 500 granzyme B substrates have been characterized and many more are expected to be identified [5]. While systematic experimental discovery
C Biomed Central

(c) 2011 Wee et al; licensee BioMed Central Ltd. This is an open access article distributed under the terms of the Creative Commons Attribution License (http://creativecommons.org/licenses/by/2.0), which permits unrestricted use, distribution, and reproduction in any medium, provided the original work is properly cited. 
and validation of bona fide substrates are necessary for elucidating the granzyme $B$ degradome, many of the processes are often time consuming and laborious. For these reasons, computational prediction of substrates could be immensely helpful in generating initial hypotheses and experimental leads. While a wide range of computational methods have been applied for substrate prediction of related proteases such as caspases [6,7], only a limited number are available for prediction of granzyme B substrates. PeptideCutter [8] is a general protease substrates cleavage prediction server which predicts for potential granyzme B cleavage sites using preferential tetrapeptide cleavage $\left(\mathrm{P}_{4}-\mathrm{P}_{3}-\mathrm{P}_{2}-\mathrm{P}_{1}\right)$ specificities derived from in vitro combinatorial library studies by Thornberry et al. [4]. Backes et al. developed the GraBCas software which extended the use of the in vitro specificities by incorporating position-specific scoring matrices and accounting for conserved residues at $\mathrm{P}_{1}$, and $\mathrm{P}_{2}$ ' positions [9]. More recently, Barkan et al. advanced the field through the application of the support vector machines (SVM) method on a set of experimentally verified cleavage sites using both sequence and structural features [10].

In this paper, we have compiled a dataset of 580 experimentally verified granzyme B cleavage sites and found distinctive patterns of residue conservation and position-specific residue propensities which could be useful for in silico prediction using machine learning algorithms. We trained a series of SVM classifiers employing Bayes Feature Extraction to predict cleavage sites using sequence windows of diverse lengths and compositions. The SVM classifiers achieved accuracy and $\mathrm{A}_{\mathrm{ROC}}$ scores between $71.00 \%$ to $86.50 \%$ and 0.78 to 0.94 respectively on independent test sets. We applied our prediction method on the Chikungunya viral proteome and identified several regulatory domains of viral proteins to be potential sites of granzyme B cleavage, suggesting direct antiviral activity of granzyme B during host-viral innate immune responses. A web server, together with reference datasets and supplementary materials, can be accessed at http://www.casbase.org/ grasvm/index.html.

\section{Results and discussion}

Sequence analysis of granzyme B cleavage sites

Using peptide combinatorial libraries, Thornberry and co-workers had previously identified the presence of distinctive sequence specificities governing protein cleavage of both caspase and granzyme B substrates [4]. In particular, specific tetrapeptide sequences upstream of the cleavage site $\left(\mathrm{P}_{4}-\mathrm{P}_{3}-\mathrm{P}_{2}-\mathrm{P}_{1}\right)$ of granzyme $\mathrm{B}$ targets serve as recognition sites for protein cleavage. The tetrapeptide "IEPD" was identified as the optimal tetrapetide cleavage sequence in vitro. However, emerging data on granzyme B substrates suggest that the in vivo cleavage specificities are far more diverse, with numerous substrates possessing cleavage specificities extending beyond the tetrapeptide sequence $[5,10]$.

We compiled a comprehensive dataset of 580 unique granzyme B cleavage sites extracted from experimentally verified substrates as reported in literature. Data was extracted from the substrates list compiled in Barkan et al. [10], as well as the proteomic studies by Van Damme et al. [5]. In addition to the $\mathrm{P}_{4} \mathrm{P}_{1}$ cleavage site sequences, segments of different lengths and compositions centered on the $\mathrm{P}_{1}$ position were selected. In all, eight groups of sequences were obtained $-\mathrm{P}_{2} \mathrm{P}_{2}, \mathrm{P}_{4} \mathrm{P}_{1}$, $\mathrm{P}_{4} \mathrm{P}_{2}{ }^{\prime}, \mathrm{P}_{4} \mathrm{P}_{4}{ }^{\prime}, \mathrm{P}_{6} \mathrm{P}_{6}, \mathrm{P}_{8} \mathrm{P}_{8}, \mathrm{P}_{10} \mathrm{P}_{10}$ ' and $\mathrm{P}_{14} \mathrm{P}_{10}$ '. We further extracted an equal number of "non-cleavage" sites by randomly selecting non-annotated tetrapeptide sequences (and other corresponding sequence segments) on the substrates. On the $\mathrm{P}_{10} \mathrm{P}_{10}$ ' dataset, we computed $\mathrm{P}_{\mathrm{x}}$ (or relative position-specific residue propensity) of each amino acid at the different residue positions along the 20-mer sequence. $P_{x}$ was computed as the ratio of the frequency of occurrence of a particular residue in the cleavage site sequences over the same residue in the non-cleavage site sequences at the particular position.

As shown in Table 1 , measurements of average $P_{x}$ in the $\mathrm{P}_{10} \mathrm{P}_{10}$ ' sequences indicate an unusually high enrichment for the negatively charged amino acids Asp and

Table 1 Average $P_{x}$ of amino acids: Average $P_{x}$ of each amino acid was calculated by averaging the $P_{x}$ values of the particular amino acid across all residue positions within the 20-mer sequence window $\left(P_{10} P_{10}\right)$

\begin{tabular}{cc}
\hline Amino acid & Average $\mathbf{P}_{\mathbf{x}}$ \\
\hline A & 1.14 \\
C & 0.72 \\
D & 1.98 \\
E & 1.46 \\
F & 0.80 \\
G & 1.02 \\
H & 0.48 \\
I & 1.05 \\
K & 0.69 \\
L & 0.88 \\
M & 1.10 \\
N & 0.86 \\
P & 0.93 \\
Q & 0.96 \\
R & 0.66 \\
S & 1.07 \\
T & 0.96 \\
V & 1.08 \\
W & 0.46 \\
Y & 0.80 \\
\hline
\end{tabular}


Glu with average $\mathrm{P}_{\mathrm{x}}$ scores of 1.98 and 1.46 respectively. Conversely, there are significantly lower propensities for the positively charged amino acids (His, Lys and Arg all possess average $P_{x}$ of less than 0.70). In addition, the large hydrophobic residue Trp is also weakly represented among the cleavage site sequences, with average $\mathrm{P}_{\mathrm{x}}$ of 0.46 . To further quantify position-specific residue propensities, we plotted a sequence logo using the $\mathrm{P}_{10} \mathrm{P}_{10}$ ' sequences and constructed a heatmap of $\mathrm{P}_{\mathrm{x}}$ scores from the same dataset (as shown in Figures 1 and 2 respectively). At $\mathrm{P}_{1}$ position, Asp is expectedly the most conserved residue, with notable presence of Glu, Asn and Ser as alternatives. Interestingly, Pro and Cys residues are more conserved in the cleavage sites compared to the non-cleavage sites at $P_{2}$ position, while $P_{3}$ is dominated by the acidic residues Asp and Glu. The $\mathrm{P}_{4}$ position showed significant propensities for the branched-chain amino acids Leu, Ile and Val. Remarkably, the most prominent feature distinguishing cleavage site sequences from non-cleavage site sequences appear to be the extended stretches of acidic residues (Asp and Glu) upstream and downstream of the cleavage site. Downstream of the cleavage site, it is further observed that small amino acids such as Gly, Ser, Ala and Leu are highly enriched at $\mathrm{P}_{1}{ }^{\prime}$ and $\mathrm{P}_{2}{ }^{\prime}$. These results indicate that cleavage sites of granzyme $B$ substrates and the flanking upstream and downstream sequences have unique position-specific residue propensities. These composite signatures could be incorporated into machine learning algorithms for the development of accurate computational prediction models.

\section{SVM prediction of granzyme B cleavage sites}

To account for these unique signatures of residue conservation and position-specific propensities for in silico prediction, we developed SVM prediction models

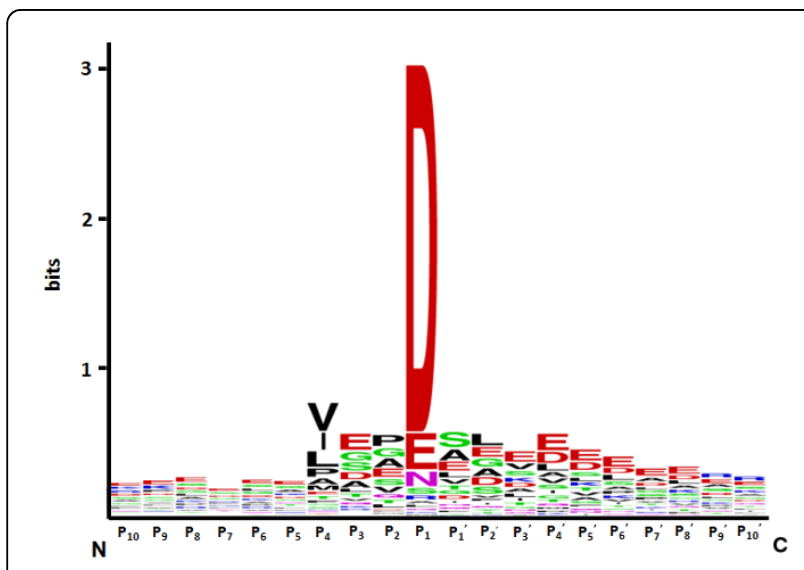

Figure 1 Sequence logo of amino acids in the vicinity of the granzyme $B$ cleavage site $\left(P_{10}\right.$ to $\left.P_{10}{ }^{\prime}\right)$

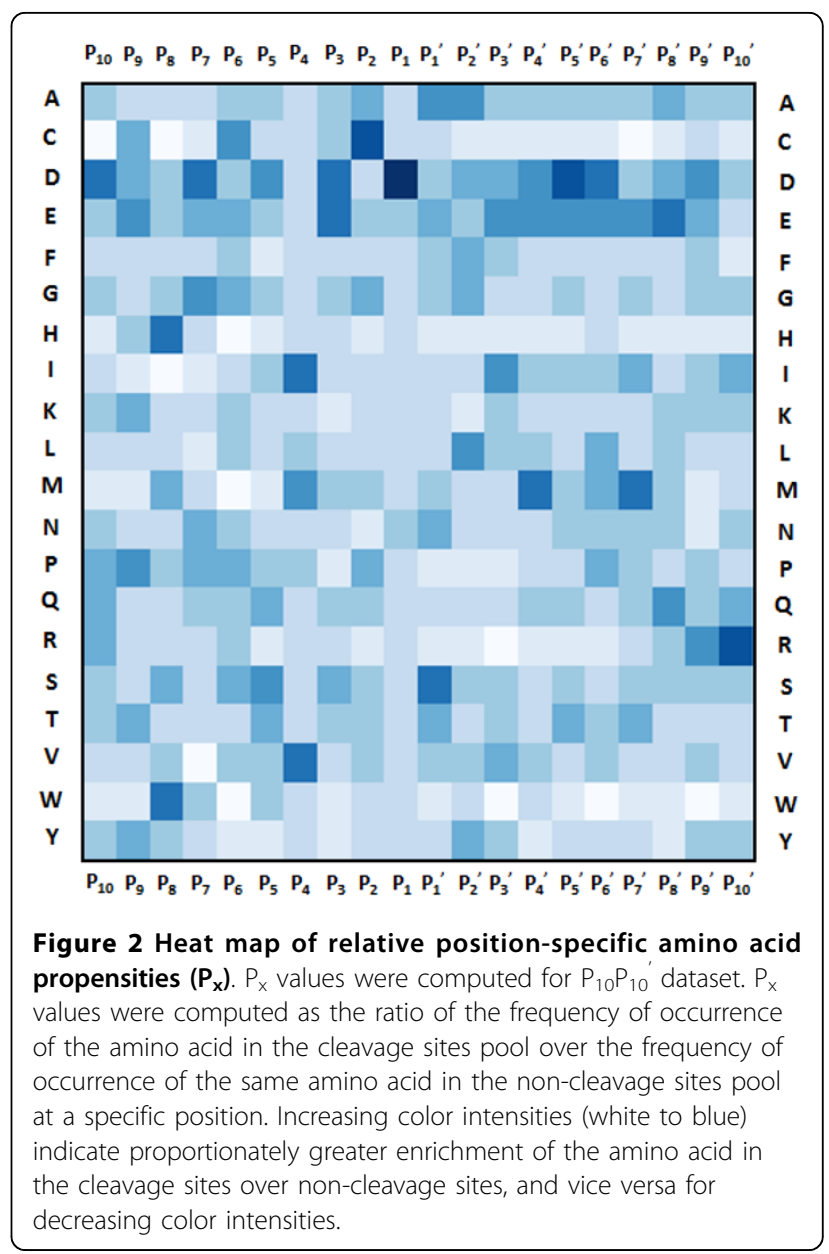

incorporating the Bayes Feature Extraction (BFE) approach as described in Shao et al.[11]. Vector representation using the BFE approach was shown to significantly improve performance in several biocomputational problems - such as the prediction of protein methylation sites [11], caspase cleavage [12] and linear B-cell epitopes [13] - over simple binary encoding schemes. In BFE, feature vectors encoded in a bi-profile manner comprising of positive position-specific and negative position-specific profiles. These profiles were generated by accounting for the frequency of occurrence of each amino acid at each position of the sequences in the positives pool (cleavage site sequences) and negatives pool (non-cleavage site sequences) respectively. Here, we trained a series of SVM classifiers on sequence windows of diverse lengths and compositions $\left(\mathrm{P}_{2} \mathrm{P}_{2}\right.$, $\mathrm{P}_{4} \mathrm{P}_{1}, \mathrm{P}_{4} \mathrm{P}_{2}, \mathrm{P}_{4} \mathrm{P}_{4}{ }^{\prime}, \mathrm{P}_{6} \mathrm{P}_{6}{ }^{\prime}, \mathrm{P}_{8} \mathrm{P}_{8}{ }^{\prime}, \mathrm{P}_{10} \mathrm{P}_{10}$ and $\left.\mathrm{P}_{14} \mathrm{P}_{10}{ }^{\prime}\right)$ using simple binary encoding and BFE schemes (details in Materials and Methods). Datasets were segmented into training and independent test sets comprising of 480 positives/480 negatives and 100 positives/100 negatives respectively. Using the RBF kernel, 10-fold cross- 
validation was implemented to acquire the optimal set of $C$ and $\gamma$ parameter values. SVM classifiers were subsequently trained on the entire training set using the optimized parameters and evaluated on the independent test sets.

As given in Table 2, the $\mathrm{P}_{4} \mathrm{P}_{1}$ classifier utilizing simple binary encoding $\left(\mathrm{P}_{4} \mathrm{P}_{1}-\mathrm{SVM}\right)$ registered an accuracy of $77.50 \%$ and $A_{R O C}$ of 0.77 on independent testing. The other classifiers observed consistent improvement in accuracy and $\mathrm{A}_{\mathrm{ROC}}$ as the sequence window extends beyond $\mathrm{P}_{4} \mathrm{P}_{1}$ to include the flanking upstream and downstream residues, achieving the best scores of $83.50 \%$ and 0.89 respectively with the $\mathrm{P}_{8} \mathrm{P}_{8}{ }^{\prime}-\mathrm{SVM}$ classifier. The $\mathrm{P}_{4} \mathrm{P}_{1}$ classifier utilizing $\mathrm{BFE}$ scheme $\left(\mathrm{P}_{4} \mathrm{P}_{1}\right.$ Bayes) attained an accuracy of $76.50 \%$ and $A_{R O C}$ of 0.84 (Table 3). In a similar fashion, prediction performance improved steadily as the sequence window is extended beyond $\mathrm{P}_{4} \mathrm{P}_{1}$, achieving the best accuracy of $86.50 \%$ with the $\mathrm{P}_{8} \mathrm{P}_{8}$-Bayes classifier and the best $\mathrm{A}_{\mathrm{ROC}}$ of 0.94 with the $\mathrm{P}_{10} \mathrm{P}_{10}$-Bayes and $\mathrm{P}_{14} \mathrm{P}_{10}$-Bayes classifiers. Interestingly, in both feature representation schemes, prediction performances did not significantly improve with sequences longer than $\mathrm{P}_{8} \mathrm{P}_{8}{ }^{\prime}$. This could be due to that fact that much of the information specific for differentiating cleavage sites from non-cleavage sites are encoded within the sequences situated closer to the cleavage sites, as evidenced by the unique residue propensities discussed earlier. In addition, accuracy and $A_{R O C}$ scores across most sequence lengths and compositions were generally higher for classifiers trained using the BFE scheme, with the greatest improvements observed when longer sequences $\left(\mathrm{P}_{6} \mathrm{P}_{6}{ }^{\prime}, \mathrm{P}_{8} \mathrm{P}_{8}, \mathrm{P}_{10} \mathrm{P}_{10}{ }^{\prime}\right.$ and $\mathrm{P}_{14} \mathrm{P}_{10}$ ') were employed.

Next, we compared our prediction method with GraBCas [9] and the SVM models developed by Barkan et al. [10]. As the GraBCas algorithm primarily focuses on the detection of specific tetrapeptide motifs, we applied the algorithm on our $\mathrm{P}_{4} \mathrm{P}_{1}$ independent test set which contains only the tetrapeptide cleavage site sequences. Using the recommended cut-off score of 0.12 , GraBCas predicted only 61 out of 100 cleavage sites correctly

Table 2 Results of SVM prediction using simple binary encoding

\begin{tabular}{|c|c|c|c|c|}
\hline SVM classifier & Sensitivity (\%) & Specificity (\%) & Accuracy (\%) & $A_{\text {ROC }}$ \\
\hline$P_{2} P_{2}^{\prime}-S V M$ & 73.00 & 68.00 & 70.50 & 0.77 \\
\hline $\mathrm{P}_{4} \mathrm{P}_{1}-\mathrm{SVM}$ & 77.00 & 78.00 & 77.50 & 0.85 \\
\hline $\mathrm{P}_{4} \mathrm{P}_{2}^{\prime}-\mathrm{SVM}$ & 85.00 & 76.00 & 80.50 & 0.89 \\
\hline $\mathrm{P}_{4} \mathrm{P}_{4}^{\prime}-\mathrm{SVM}$ & 84.00 & 80.00 & 82.00 & 0.89 \\
\hline$P_{6} P_{6}^{\prime}-S V M$ & 84.00 & 82.00 & 83.00 & 0.89 \\
\hline$P_{8} P_{8}^{\prime}-S V M$ & 83.00 & 84.00 & 83.50 & 0.89 \\
\hline $\mathrm{P}_{10} \mathrm{P}_{10}^{\prime}-\mathrm{SVM}$ & 81.00 & 82.00 & 81.50 & 0.89 \\
\hline $\mathrm{P}_{14} \mathrm{P}_{10}{ }^{\prime}-\mathrm{SVM}$ & 78.00 & 81.00 & 79.50 & 0.88 \\
\hline
\end{tabular}

Table 3 Results of SVM prediction using Bayes Feature Extraction

\begin{tabular}{|c|c|c|c|c|}
\hline SVM classifier & Sensitivity (\%) & Specificity (\%) & Accuracy (\%) & $A_{\mathrm{ROC}}$ \\
\hline $\mathrm{P}_{2} \mathrm{P}_{2}^{\prime}$-Bayes & 71.00 & 71.00 & 71.00 & 0.78 \\
\hline $\mathrm{P}_{4} \mathrm{P}_{1}$-Bayes & 79.00 & 74.00 & 76.50 & 0.84 \\
\hline $\mathrm{P}_{4} \mathrm{P}_{2}^{\prime}$-Bayes & 82.00 & 80.00 & 81.00 & 0.89 \\
\hline P4P4'-Bayes & 82.00 & 81.00 & 81.50 & 0.91 \\
\hline$P_{6} P_{6}^{\prime}$-Bayes & 86.00 & 84.00 & 85.00 & 0.91 \\
\hline $\mathrm{P}_{8} \mathrm{P}_{8}^{\prime}$-Bayes & 89.00 & 84.00 & 86.50 & 0.93 \\
\hline $\mathrm{P}_{10} \mathrm{P}_{10}$-Bayes & 87.00 & 85.00 & 86.00 & 0.94 \\
\hline $\mathrm{P}_{14} \mathrm{P}_{10}{ }^{\prime}$-Bayes & 88.00 & 82.00 & 85.00 & 0.94 \\
\hline
\end{tabular}

$\left(S_{n}=61 \%\right)$. On the same dataset, our $\mathrm{P}_{4} \mathrm{P}_{1}-\mathrm{SVM}$ and $\mathrm{P}_{4} \mathrm{P}_{1}$-Bayes classifiers respectively predicted 77 out of $100\left(S_{n}=77 \%\right)$ and 79 out of $100\left(S_{n}=79 \%\right)$ cleavage sites correctly. The weaker sensitivity scores observed for GraBCas could be due to the utilization of position-specific scoring matrices (PSSMs) which are derived from a small, out-dated set of in vitro cleavage specificities and the absolute requirement of Asp residue at $\mathrm{P}_{1}$ on the cleavage sites. To further evaluate the performance of the PSSM-based algorithm in our context, we constructed PSSMs derived from our entire dataset of cleavage sites, and found that the $A_{R O C}$ scores of the PSSMbased predictors were generally poorer than our SVMbased classifiers (data not shown). In Barkan et al., the best SVM classifier recorded a true positive rate (TPR) of 0.79 and false positive rate (FPR) of 0.21 at the critical point on the receiver operating characteristic (ROC) curve when tested on an independent test set. In our SVM method, several classifiers encoded using the BFE scheme registered better prediction performance when measured by the same metrics; $\mathrm{P}_{10} \mathrm{P}_{10}$-Bayes with TPR of 0.86 and FPR of 0.14 , as well as $\mathrm{P}_{14} \mathrm{P}_{10}$-Bayes, $\mathrm{P}_{8} \mathrm{P}_{8}{ }^{\prime}$ Bayes and $\mathrm{P}_{6} \mathrm{P}_{6}$-Bayes with TPRs of 0.85 and FPRs of 0.15 .

\section{Prediction of granzyme B cleavage of CHIKV proteome}

To investigate the applicability of our computational method, we applied the SVM classifiers on the proteome of the Chikungunya virus (CHIKV) and analyzed for the presence of hitherto undiscovered granzyme $\mathrm{B}$ cleavage sites. CHIKV is a member of the alphavirus family and has been known to be transmitted to humans via the bite of the virus-borne Aedes mosquito [14]. Acute infection of CHIKV results in symptoms such as abrupt fever, skin rash and arthralgia. As CHIKV epidemics have been re-emerging in recent times, there have been concerted efforts directed toward developing relevant vaccines and drug therapies. During viral infections, granzyme B has been reported to mediate downstream cleavage of critical host regulatory proteins, leading to the induction of the apoptotic cell death, and hence 
disruption of viral propagation [15]. Although granzyme B-induced apoptotic cell death has long been considered the de facto mechanism for killing virus-infected cells, emerging evidence suggest that the enzyme could exert direct antiviral activity through cleavage of the viral proteins [15]. For these reasons, it is intuitive to speculate if the CHIKV proteome may be directly regulated by granzyme $\mathrm{B}$ activity in this manner and if cleavage of specific CHIKV proteins will potentiate the host innate immune responses against viral infectivity.

Four non-structural and four structural proteins of the CHIKV proteome (strain: LR2006_OPY1) were predicted for granzyme $\mathrm{B}$ cleavage sites using the $\mathrm{P}_{8} \mathrm{P}_{8}$ '-Bayes classifier. Since the majority of experimentally verified cleavage sites were known to be cleaved after the Asp residue, we have restricted our prediction scans to only cleavage sites containing Asp residue at $\mathrm{P}_{1}$. As shown in Table 4, we found potential granzyme $B$ cleavage sites in all CHIKV proteins except the structural proteins E1, E3 and 6K. A significantly larger proportion of these sites were found in the non-structural proteins NSP1, NSP2, NSP3 and NSP4, as compared to the structural proteins E2 and capsid. As the alphaviral non-structural proteins are known to be involved in viral survival and replication, we would expect the cleavage of these proteins by granzyme B to abrogate viral survival mechanisms at different points of the viral reproduction cycle [16]. Indeed, the cleavage of NSP1 protein at Asp-11 and Asp-58, which are both localized within the methyltransferase domain, could lead to inhibition of the mRNA capping during RNA synthesis. Conversely, the cleavage of NSP2 helicase domain at Asp-247 and Asp343, as well as the RNA polymerase domain at Asp-291, Asp-371, Asp-476 and Asp-540 on the NSP4 protein could hinder viral RNA synthesis and translation. In addition, cleavage of the capsid protein at Asp-112 and Asp-114 within the protease domain might lead to prevention of auto-cleavage of the immature capsid protein from the viral structural polyprotein.

\section{Conclusions}

In this paper, we constructed a comprehensive database of experimentally verified granzyme B cleavage sites for analysis and development of prediction methods. We discovered that flanking sequences of cleavage sites possess distinctive residue composition and position-specific propensity patterns which could be helpful in discriminating the cleavage sites from non-cleavage sites in silico. We have rigorously tested SVM classifiers employing simple binary encoding and the Bayes Feature Extraction schemes to predict granzyme B cleavage sites. Results also show that the best classifiers are more effective than existing algorithms. We applied our prediction method on the Chikungunya viral proteome and identified several regulatory domains of viral proteins to be potential targets of granzyme B cleavage, suggesting a direct antiviral function of granzyme B during host-viral innate immune responses. To complement experimental research, we have implemented our prediction method on a web server which is freely accessible at http://www. casbase.org/grasvm/index.html. In the immediate future, we will be exploring the influence of cleavage site secondary structures, solvent accessibilities and other physicochemical properties on protease-substrate cleavage specificities, as well as their potential for enhancing the performance of our SVM prediction models. Computational prediction of granzyme B substrates will complement on-going experimental efforts and refine our understanding of the biochemistry of this fascinating protease and its relatives.

\section{Materials and methods \\ Datasets}

We extracted a pool of 779 unique, experimentally verified cleavage sites from literature. 723 sequences were derived from proteomic experimental studies conducted by Van Damme et al. [5], with the remaining 56 from systematic in vitro and in vivo experiments as compiled in Barkan et al. [10]. We further extracted sequence

Table 4 Prediction of granzyme B cleavage of CHIKV proteome

\begin{tabular}{|c|c|c|}
\hline Protein & Biological activity and function & Cleavage sites* \\
\hline NSP1 & Non-structural: mRNA capping & $\underline{9}, 11, \underline{58}, 525$ \\
\hline NSP2 & Non-structural: NTPase, helicase and protease activities & $116, \underline{247}, \underline{343}$ \\
\hline NSP3 & Non-structural: ADP-ribose phosphatase activity & $181, \overline{350}, \overline{363}, 506$ \\
\hline NSP4 & Non-structural: RNA polymerase activity & $219, \underline{371}, \underline{476}, \underline{540}$ \\
\hline E1 & Structural: virus-host cell fusion & $\overline{\mathrm{Nil}}--$ \\
\hline E2 & Structural: virus-host cell attachment & 77 \\
\hline E3 & Structural: unknown & $\mathrm{Nil}$ \\
\hline Capsid & Structural: protease, viral nucleocapsid formation & $\underline{112}, \underline{174}$ \\
\hline $6 \mathrm{~K}$ & Structural: membrane permeabilization, budding of viral particles & $\overline{\mathrm{Nil}}$ \\
\hline
\end{tabular}

*Position of the $\mathrm{P}_{1}$ residue on the substrate. All predicted cleavage sites contain Asp at $\mathrm{P}_{1}$. Underlines indicate $\mathrm{P}_{1}$ location in the functional domain(s) of protein. 
segments of different lengths flanking the $\mathrm{P}_{1}$ cleavage sites. In all, eight datasets were constructed: the tetrapeptide cleavage site sequences (referred to as $\mathrm{P}_{4} \mathrm{P}_{1}$ dataset) and sequences containing residues extended to $\mathrm{P}_{14}$ and $\mathrm{P}_{10}{ }^{\prime}\left(\mathrm{P}_{2} \mathrm{P}_{2}^{\prime}, \mathrm{P}_{4} \mathrm{P}_{2}^{\prime}, \mathrm{P}_{4} \mathrm{P}_{4}^{\prime}, \mathrm{P}_{6} \mathrm{P}_{6}^{\prime}, \mathrm{P}_{8} \mathrm{P}_{8}^{\prime}, \mathrm{P}_{10} \mathrm{P}_{10}{ }^{\prime}\right.$ and $\mathrm{P}_{14} \mathrm{P}_{10}$ ' datasets). These sequences were assigned as positive examples for analysis as well as for development of the SVM method. An equal number of "non-cleavage sites" or negative examples were obtained by randomly extracting $\mathrm{P}_{1}$ residues on the substrates. Sequence segments of the aforementioned lengths and compositions were obtained as detailed earlier. All datasets of positive and negative sequences (779/779) were subsequently subjected to homology filtering using the CD-HIT clustering algorithm [17] where sequences bearing more than $85 \%$ sequence identity with any other sequence in the dataset were eliminated. The final datasets comprised of 580 positive and 580 negative sequences (the complete list of cleavage sites is available in Additional File 1). For analysis, all 580 positives and 580 negatives from the $\mathrm{P}_{10} \mathrm{P}_{10}$ ' dataset were used. For SVM model development, datasets were partitioned into training and test sets consisting of 480 positives/480 negatives and 100 positives/100 negatives respectively.

\section{Sequence analysis}

The relative position-specific residue propensity $P_{x}$ was computed as the ratio of the frequency of occurrence of a particular amino acid in the cleavage sites pool to its frequency of occurrence in the non-cleavage sites pool at a specific position on the sequence. Using the $\mathrm{P}_{10} \mathrm{P}_{10}$, dataset, $\mathrm{P}_{\mathrm{x}}$ scores were calculated for every amino acid at each of the twenty residue positions and visualized on heat maps. Additionally, we constructed a sequence logo, representation of the positive sequences from the $\mathrm{P}_{10} \mathrm{P}_{10}$, dataset using WebLogo [18].

\section{SVM vector representation}

To encapsulate sequence information for SVM training and testing, input vectors were constructed using simple binary or bi-profile Bayes Features encoding. For simple binary encoding, each amino acid is represented by a vector of 20 dimensions, comprising of binary values of zeroes and ones. For example, alanine was represented as $[0,0,0,0,0,0,0,0,0,0,0,0,0,0,0,0,0,0,0,1]$ and cysteine as $[0,0,0,0,0,0,0,0,0,0,0,0,0,0,0,0,0,0,1,0]$. Hence, in this case, a 20-mer sequence will be represented by a vector of 400 dimensions ( $20 \times 20)$. Detailed description on bi-profile vector encoding using Bayes Features is available in Shao et al. [11]. In short, feature vectors contain information from both positive position-specific and negative position-specific profiles. These profiles were generated by accounting for the frequency of occurrence of each amino acid at each position of the sequences in the positives pool (cleavage site sequences) and negatives pool (non-cleavage site sequences) respectively. Therefore, a 20 -mer sequence (from the $\mathrm{P}_{10} \mathrm{P}_{10}$ ' dataset) would be represented by a feature vector of 40 dimensions $(20 \mathrm{x}$ 2), containing information of the residues in both positive (cleavage site sequences) and negative (non-cleavage site sequences) spaces. For all sequence representations, $\mathrm{P}_{1}$ residues were excluded from the feature vectors.

\section{SVM model development}

To train and test the SVM models, we used the LIBSVM package provided by Chang and Lin [19]. For details on the SVM method, readers are advised to consult the article by Burges [20]. In short, SVM is grounded on the structural risk minimization concept from statistical learning theory. A set of training examples (positives and negatives) can be encoded by the feature vectors $x_{\mathrm{i}}(i=1,2, \ldots \mathrm{N})$ with resultant classes $y_{i} \in\{+1,-1\}$. The SVM algorithm trains a classifier by representing the input feature vectors, using a kernel function in the majority of cases, onto a high-dimensional space, and then selects a discriminating hyperplane that separates the two classes with maximal margin and the least error. The decision function for classification of unseen examples is defined as:

$$
f(x)=\operatorname{sign}\left(\sum_{i=1}^{N} \alpha_{i} y_{i} K\left(x_{i} \cdot x_{j}\right)+b\right)
$$

where $\mathrm{K}\left(x_{i} \cdot x_{j}\right)$ is the kernel function, and the parameters are resolved by maximizing the following:

$$
\sum_{i=1}^{N} \alpha_{i}-\frac{1}{2} \sum_{i=1}^{N} \sum_{j=1}^{N} \alpha_{i} \alpha_{j} y_{i} y_{j} K\left(x_{i} \cdot x_{j}\right)
$$

with the following constraints:

$$
\sum_{i=1}^{N} \alpha_{i} y_{i}=0 \text { and } 0 \leq \alpha_{i} \leq C
$$

$C$ is the regularization variable that directs the tradeoff between margin and classification error. We used the radial basis function (RBF) kernel and performed grid-based optimization for $\gamma$, which controls the capacity of the RBF kernel, and $C$ using 10 -fold cross-validation. In 10-fold cross-validation, the training set was randomly partitioned into ten subsets where one of the subsets was used as the test set while the other subsets were used for training the classifier. The trained classifier was evaluated using the test set. This procedure was repeated ten times using different subsets for testing, hence making sure that all subsets were utilized for both training and testing. The optimized $\gamma$ and $C$ values 
were applied towards training the entire training set to generate the SVM classifier for independent testing on an out-of-sample test set. Graphical plots of optimization results are provided in Additional File 2.

\section{Evaluation of model performance}

A set of statistical variables were established to evaluate the performance of the SVM classifier for the prediction of granzyme B cleavage sites:

(i) True Positives (TP), for the number of correctly classified cleavage sites.

(ii) False Positives $(F P)$, for the number of incorrectly classified non-cleavage sites.

(iii) True Negatives $(T N)$, for the number of correctly classified non-cleavage sites.

(iv) False Negatives $(F N)$, for the number of incorrectly classified cleavage sites.

Sensitivity $\left(S_{n}\right)$ and Specificity $\left(S_{p}\right)$, which measures the capability of the model to correctly classify the cleavage sites and non-cleavage sites respectively, were computed as well:

$$
\begin{aligned}
& S_{n}=\frac{T P}{T P+F N} \\
& S_{p}=\frac{T N}{T N+F P}
\end{aligned}
$$

To measure the overall model performance, we computed Accuracy $\left(A_{c c}\right)$ :

$$
A_{c c}=\frac{T P+T N}{T P+F N+T N+F P}
$$

In addition, we plotted the receiver operating characteristic curve (ROC) and computed the area under the curve $\left(\mathrm{A}_{\mathrm{ROC}}\right)$ for threshold independent evaluation. To compare against the prediction model developed by Barkan et al., we further determined the critical points on the ROCs of our SVM classifiers, which are defined as the points where the ROC curves intersect the lines connecting coordinates $(1,0)$ and $(0,1)$ on the graphs.

\section{Additional material}

Additional File 1: Dataset of granzyme B cleavage sites This file contains the dataset of granzyme B cleavage sites. Training and test set sequences are listed on different tabs.

Additional File 2: SVM parameter optimization Training sets of different sequence window datasets were trained under 10-fold crossvalidation using various combinations of $C$ and $\gamma$ values. The optimal $C$ and $\gamma$ values for each training set are used to train the final SVM classifier. Optimal values are indicated below the corresponding chart.

\section{Acknowledgements}

This study was sponsored by a research grant from the Joint Council Office (JCO) of A*STAR Singapore.

This article has been published as part of BMC Genomics Volume 12 Supplement 3, 2011: Tenth International Conference on Bioinformatics - First ISCB Asia Joint Conference 2011 (InCoB/ISCB-Asia 2011): Computational Biology. The full contents of the supplement are available online at http:// www.biomedcentral.com/1471-2164/12?issue=S3.

\section{Author details}

1 Data Mining Department, Institute for Infocomm Research, 1 Fusionopolis Way, \#21-01 Connexis South Tower, Singapore 138632. ${ }^{2}$ Singapore Immunology Network, 8A Biomedical Grove, \#04-06 Immunos, Biopolis, Singapore 138648. ${ }^{3}$ Department of Biochemistry, Yong Loo Lin School of Medicine, National University of Singapore, 8 Medical Drive, Singapore 117597.

\section{Authors' contributions}

LJKW conceptualized the study and managed the technical aspects of the project. EPSE assisted with the preparation of data and analysis of results. LFPN and JCT contributed with ideas and assisted with the manuscript preparation. All authors read and approved the final manuscript.

\section{Competing interests}

The authors declare that they have no competing interests.

Published: 30 November 2011

\section{References}

1. López-Otín C, Overall CM: Protease degradomics: a new challenge for proteomics. Nat Rev Mol Cell Biol 2002, 3:509-519.

2. Chowdhury D, Lieberman J: Death by a thousand cuts: granzyme pathways of programmed cell death. Annu Rev Immunol 2008, 26:389-420.

3. Los M, Stroh C, Janicke RU, Engels $1 H$, Schulze-Osthoff K: Caspases: more than just killers? Trends Immunol 2001, 22:31-34.

4. Thornberry NA, Rano TA, Peterson EP, Rasper DM, Timkey T, Garcia-Calvo M, Houtzager VM, Nordstrom PA, Roy S, Vaillancourt JP, Chapman KT, Nicholson DW: A combinatorial approach defines specificities of members of the caspase family and granzyme B. Functional relationships established for key mediators of apoptosis. J Biol Chem 1997, 272:17907-11.

5. Van Damme P, Maurer-Stroh S, Plasman K, Van Durme J, Colaert N, Timmerman E, De Bock PJ, Goethals M, Rousseau F, Schymkowitz J, Vandekerckhove J, Gevaert K: Analysis of protein processing by N-terminal proteomics reveals novel species specific substrate determinants of granzyme B orthologs. Mol Cell Proteomics 2009, 8:258-72.

6. Wee LJ, Tan TW, Ranganathan S: SVM-based prediction of caspase substrate cleavage sites. BMC Bioinformatics 2006, 7(Suppl 5):S14.

7. Piippo M, Lietzén N, Nevalainen OS, Salmi J, Nyman TA: Pripper: prediction of caspase cleavage sites from whole proteomes. BMC Bioinformatics 2010, 11:320.

8. Gasteiger E, Hoogland C, Gattiker A, Duvaud S, Wilkins MR, Appel RD, Bairoch A: Protein Identification and Analysis Tools on the ExPASy Server. In The Proteomics Protocols Handbook. Humana Press; Walker JM 2005:571-607.

9. Backes C, Kuentzer J, Lenhof HP, Comtesse N, Meese E: GraBCas: a bioinformatics tool for score-based prediction of Caspase- and Granzyme B-cleavage sites in protein sequences. Nucleic Acids Res 2005, 33(Web server issue):W208-W213.

10. Barkan DT, Hostetter DR, Mahrus S, Pieper U, Wells JA, Craik CS, Sali A: Prediction of protease substrates using sequence and structure features. Bioinformatics 2010, 26:1714-1722.

11. Shao J, Xu D, Tsai SN, Wang Y, Ngai SM: Computational identification of protein methylation sites through bi-profile Bayes feature extraction. PloS One 2009, 4:e4920.

12. Song J, Tan H, Shen H, Mahmood K, Boyd SE, Webb Gl, Akutsu T, Whisstock JC: Cascleave: towards more accurate prediction of caspase substrate cleavage sites. Bioinformatics 2010, 6:752-760. 
13. Wee LJ, Simarmata D, Kam YW, Ng LF, Tong JC: SVM-based prediction of linear B-cell epitopes using Bayes feature extraction. BMC Genomics 2010, 11(Suppl 4):S21.

14. Pialoux $G$, Gauzère BA, Jauréguiberry S, Storbel M: Chikungunya, an epidemic arbovirosis. Lancet 2007, 7:319-327.

15. Romero V, Andrade F: Non-apoptotic functions of granzymes. Tissue Antigens 2008, 71:409-416.

16. Kam YW, Ong EK, Rénia L, Tong JC, Ng LFP: Immuno-biology of Chikungunya and implications for disease intervention. Microbes Infect 2009, 11:1186-1196.

17. Huang Y, Niu B, Gao Y, Fu L, Li W: CD-HIT Suite: a web server for clustering and comparing biological sequences. Bioinformatics 2010, 26:680-682.

18. Crooks GE, Hon G, Chandonia JM, Brenner SE: WebLogo: a sequence logo generator. Genome Res 2004, 14:1188-1190.

19. Chang CC, Lin CJ: LIBSVM: a library for support vector machines.[http:// www.csie.ntu.edu.tw/ cjlin/libsvm].

20. Burges CJC: A tutorial on support vector machines for pattern recognition. Data Mining and Knowledge Discovery 1998, 2:121-167.

doi:10.1186/1471-2164-12-S3-S11

Cite this article as: Wee et al:: In silico prediction of the granzyme B degradome. BMC Genomics 2011 12(Suppl 3):S11.

\section{Submit your next manuscript to BioMed Central} and take full advantage of:

- Convenient online submission

- Thorough peer review

- No space constraints or color figure charges

- Immediate publication on acceptance

- Inclusion in PubMed, CAS, Scopus and Google Scholar

- Research which is freely available for redistribution

Submit your manuscript at www.biomedcentral.com/submit 